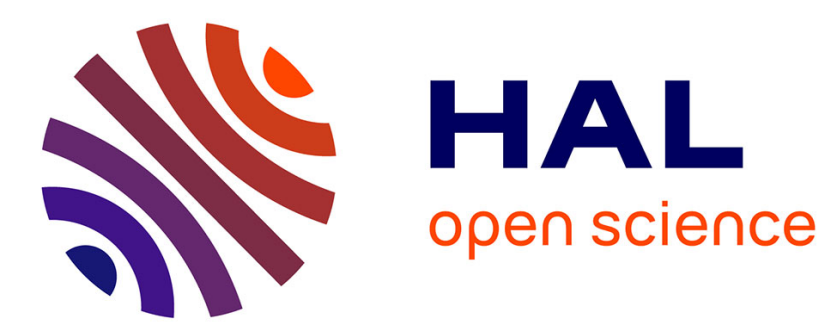

\title{
Chromatic analysis of harmonic Fresnel lenses by FDTD and angular spectrum methods
}

\author{
Jianming Yang, Patrice Twardowski, Philippe Gérard, Wenhui Yu, Joël \\ Fontaine
}

\section{- To cite this version:}

Jianming Yang, Patrice Twardowski, Philippe Gérard, Wenhui Yu, Joël Fontaine. Chromatic analysis of harmonic Fresnel lenses by FDTD and angular spectrum methods. Applied optics, 2018, 57 (19), pp.5281-5287. 10.1364/AO.57.005281 . hal-02054648

\section{HAL Id: hal-02054648 https://hal.science/hal-02054648}

Submitted on 1 Mar 2019

HAL is a multi-disciplinary open access archive for the deposit and dissemination of scientific research documents, whether they are published or not. The documents may come from teaching and research institutions in France or abroad, or from public or private research centers.
L'archive ouverte pluridisciplinaire HAL, est destinée au dépôt et à la diffusion de documents scientifiques de niveau recherche, publiés ou non, émanant des établissements d'enseignement et de recherche français ou étrangers, des laboratoires publics ou privés. 


\title{
Chromatic analysis of harmonic Fresnel lenses by FDTD and angular spectrum methods
}

\author{
Jianming Yang, ${ }^{1,2, *}$ Patrice TWARdoWski, ${ }^{1}$ Philippe Gérard, ${ }^{1}$ Yu Wenhui ${ }^{1,2}$ And \\ JOËL FONTAINE ${ }^{1}$ \\ ${ }^{1}$ ICube UMR-7357, CNRS, University of Strasbourg, INSA, Strasbourg-IIIkirch, France \\ ${ }^{2}$ Changchun Institute of Optics, Fine Mechanics and Physics, Chinese Academy of Sciences, Changchun, Jilin 130033, China \\ *Corresponding author: jianming.yang@unistra.fr \\ Received XX Month XXXX; revised XX Month, XXXX; accepted XX Month XXXX; posted XX Month XXXX (Doc. ID XXXXX); published XX Month XXXX
}

\begin{abstract}
In this paper, we present a detailed and rigorous study of cylindrical harmonic Fresnel lenses (HFLs) using finite difference time domain method (FDTD) and angular spectrum method (ASM). The HFL is a kind of diffractive lens that can have maximum diffraction efficiency at several discrete harmonic wavelengths, which is suitable for some broadband applications. Previous studies on HFLs were investigated mainly in the domain of paraxial approximation. By using our proposed calculation method, we have determined the efficiency, focal length, maximum focus intensity and full width at half maximum (FWHM) of the focal spot for several harmonic numbers and for F-numbers of $0.5,1$ and 3 . To compare with the paraxial approximation, we have presented the response to both $s$-polarized and $p$-polarized light with constant refractive index and real dispersive material BK7. Moreover, we have also analyzed the cases with oblique illumination. We have shown that the harmonic wavelengths do not change with F/\# and that the diffraction efficiency and FWHM of the focus increase as F/\# increases. New results on harmonic wavelengths shift and oblique angle of incidence response have been detailed. (C) 2018 Optical Society of America
\end{abstract}

OCIS codes: (050.1965) Diffractive lenses; (050.1755) Computational electromagnetic methods,

http://dx.doi.org/10.1364/AO.99.099999

\section{INTRODUCTION}

The diffractive Fresnel lens (DFL), also called kinoform, was firstly invented by a research group of IBM [1, 2]. Since then, tremendous interests have been aroused due to its compactness. However, the dispersion of a DFL is large compared to optical glass (Abbe number of -3.5), excluding direct broadband application. As its dispersion sign is opposite to the normal optical glass, hybrid diffractive-refractive achromatic lenses have been studied and used [3]. The optical power of the diffractive element is chosen much lower than the refractive one. These kinds of structures can reduce the dimension and weight of optical systems. In order to achromatize by only using diffractive optical elements (DOEs), two main paths have been proposed: stacks of DOEs with different Abbe numbers and harmonic lenses. However, the stack of DOEs can only increase the broadband efficiency. The Abbe number is still around -3.5 [4-8]. A harmonic Fresnel lens (HFL) or multi-order diffractive lens can keep high diffraction efficiency and constant focal length at several discrete wavelengths $[9,10]$. Recently, metasurfaces have been proposed to function as flat lenses, which paves a new way for optical components [11-15]. However, the mass manufacturing for this kind of nanostructure is still challenging.

With broadband properties, HFLs can be applied in many situations, such as intraocular lenses [16], contact lenses and optical detection in microflow cytometry [17]. The previous works focused on HFLs have been carried out with scalar approach $[9,10,18]$. The results become inaccurate when $\mathrm{F} / \#$ is smaller than 1 [19]. Based on the boundary element method (BEM) or the improved first Rayleigh-Sommerfeld method (IRSM), metallic cylindrical micro mirrors or cylindrical Fresnel lenses have been deeply studied [19-26]. However, to our knowledge, rigorous studies of harmonic Fresnel lenses (HFLs) has not been performed for the moment. Compared to ordinary Fresnel lenses, the broadband property of HFL is more effective.

The finite difference time domain (FDTD) is very suitable to calculate the broadband properties as only one simulation that covers the selected spectrum is needed. To reduce the computational time, the height of the FDTD region is limited to nearly the same height as the HFLs. For the field outside the HFLs, as the medium is homogeneous, we can use the angular spectrum method (ASM) to calculate the field [27-30]. In this paper, we apply FDTD plus ASM to calculate the rigorous properties of HFLs. To reduce the calculation burden, we consider two-dimensional cases which correspond to cylindrical lenses. Different $\mathrm{F} / \#$ are considered under $\mathrm{p}$ and $\mathrm{s}$ polarized illuminations. Results with on-axis and off-axis illuminations are also presented. 


\section{BASIC PRINCIPLE OF HFLS}

Based on scalar diffraction theory and paraxial approximation condition, the maximum effective thickness of an ordinary Fresnel lens is [2]:

$$
h_{\max }=\frac{\lambda_{d}}{n-1},
$$

where $\lambda_{d}$ is the design wavelength and $n$ is the refractive index of the lens material. For HFL with a harmonic number $p_{h}$, the thickness is $[9$, 10]:

$$
h_{\max }=p_{h} \frac{\lambda_{d}}{n-1} .
$$
10]:

The harmonic wavelengths are a set of discrete values given by $[9$,

$$
\lambda_{p}=\frac{p_{h} \lambda_{d}}{m}
$$

where $m$ is a positive integer that equals the diffractive order. In the case of the paraxial approximation, the dependence of the focal length with the illumination wavelength $\lambda$ can be expressed as [10]:

$$
f(\lambda)=\frac{p_{h} \lambda_{d} f_{d}}{m \lambda},
$$

where $f_{d}$ is the aimed focal length.

The scalar diffraction efficiency, $\eta_{m}$, of the $m$ th diffracted order is given by $[9,10]$ :

$$
\eta_{m}=\sin c^{2}\left\{\frac{p_{h} \lambda_{d}[n(\lambda)-1]}{\lambda\left[n\left(\lambda_{d}\right)-1\right]}-m\right\}
$$

The diffraction efficiency $\eta_{m}$ equals to 1 when $\lambda$ satisfies Eq. (3), neglecting reflectance and assuming a constant index $n$ in the visible spectral range.

An ordinary Fresnel lens corresponds to a HFL when $p_{h}=1$. As $p_{h}$ increases, the height of the lens becomes higher and each groove becomes larger. Fig. 1 shows this effect.

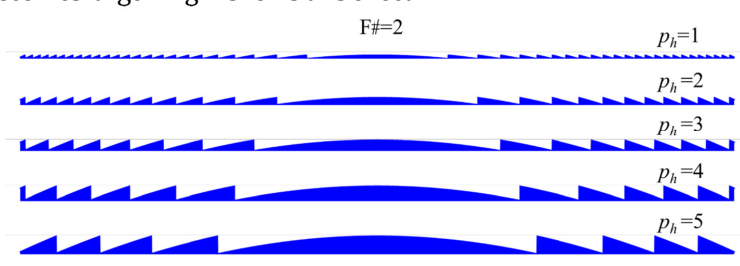

Fig. 1. Modification of the height and width of grooves when the harmonic number of a HFL increases, with a constant $F / \#=2$. The diameter is $400 \mu \mathrm{m}$.

Figure 2 shows a detailed cross-section of a HFL. Two grooves with rotational symmetry are presented. In this paper, we define the grooves as a phase shift of $2 p_{h} \pi$. Point 0 is the center of the groove numbered 0 which is defined by the semi-section $\mathrm{OA}^{\prime}$. Points A and B' define the groove numbered 1 . To obtain a constructive interference, the radius of the first groove should be:

$$
r_{1}=\sqrt{2 f p_{h} \lambda_{d}+\left(p_{h} \lambda_{d}\right)^{2}} .
$$

By generalization, the radius of a groove numbered $i$ is given by:

$$
r_{i}=\sqrt{2 i f_{d} p_{h} \lambda_{d}+\left(i p_{h} \lambda_{d}\right)^{2}} .
$$

Suppose $O$ is the origin of coordinates, according to Fermat principle, we have $P F=n P^{\prime} O+O F$, where $P$ is an arbitrary point on the profile. Hence, the equation of the profile in each groove is given by:

$$
x_{i}(r)=\frac{f_{d}-n \sqrt{f_{d}^{2}+r_{i}^{2}}+\sqrt{f_{d}^{2}\left(1+n^{2}\right)+\left(n^{2}-1\right) r^{2}+r_{i}^{2}-2 n f_{d} \sqrt{f_{d}^{2}+r_{i}^{2}}}}{1-n^{2}},
$$

which is a part of a hyperbola and where $r=\sqrt{y^{2}+z^{2}}$. The maximum groove depth decreases as the groove number increases, which means A'A>B'B in Fig. 2.

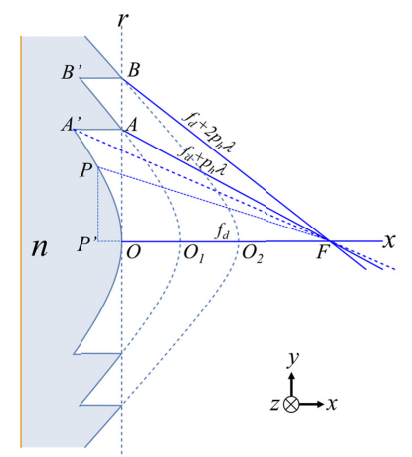

Fig. 2. Principle of a harmonic lens with a harmonic number equals $p_{h}$.

A conventional perfect refractive lens composed of one plane surface and one hyperbola can focus perfectly a normal incident plane wave on the image focal point. The asymptotic line of this hyperbola determines the minimum $\mathrm{F} / \#$ which is given by:

$$
F / \#_{\min }=\frac{1}{2 \sqrt{n^{2}-1}} .
$$

For a Fresnel lens, each groove numbered $i$ is a hyperbola with a different vortex $O_{i}$ (see Fig. 2 with the grooves 0,1 and 2). Physically, the vortices should not overpass the image focal point $F$, which determines the minimum F/\# of a HFL. The vortex coincides with F when:

$$
r_{i} \geq f \sqrt{n^{2}-1},
$$

which gives the same minimum $\mathrm{F} / \#$ as $\mathrm{Eq}$ (8). As an example, if $\mathrm{n}=1.5$, the minimum $F / \#$ is 0.45 . The physical origin of this $F / \#$ limitation is the critical angle on the profile inside the lens material. Light will be totally reflected if the $\mathrm{F} / \#$ is smaller than this limitation.

\section{OPTICAL PROPERTIES OF HFL WITH NORMAL ILLUMINATION}

For all the simulations, the electromagnetic near-field distribution is obtained numerically by the commercially available tool, namely, FDTD solutions from Lumerical. We use a maximum mesh size of $\lambda /(15 n)$, where $n$ is the refractive index of the corresponding medium. We apply perfect matched layers (PMLs) as the boundary conditions in the four sides. All the simulations are performed in 2D. For the computation of the field distribution in the air we use ASM. Figure 3 shows an example of outputs from FDTD and ASM field projection for a HFL with $p_{h}=9, \mathrm{~F} / \#=1$, the diameter of the lens is $400 \mu \mathrm{m}$ and $\lambda_{d}=0.5$ $\mu \mathrm{m}$. The illumination wavelength is also $0.5 \mu \mathrm{m}$. In the left part, we 
show the real value of the electric field (s polarized) inside the HFL. In the right part, the intensity distribution of the electric field is given with the position of the focal image area. We get a focusing at $x$ coordinate of $400 \mu \mathrm{m}$. The full width at half maximum (FWHM) of the focal spot is $0.5 \mu \mathrm{m}$ and the axial depth of focus is $6 \mu \mathrm{m}$ (the distance between the position of intensity equals $I_{\max } / e$-on the right side and left side).

Several HFLs with the same diameter $D=400 \mu \mathrm{m}$ but different $\mathrm{F} / \#$ have been investigated below. The design wavelength is $\lambda_{d}=0.5 \mu \mathrm{m}$ and the calculated spectral range is from 0.4 to $0.7 \mu \mathrm{m}$ with a step size of $0.001 \mu \mathrm{m}$, which covers the visible spectrum. At the beginning and for the sake of simplicity, we have supposed that the refractive index of the HFL is $n_{d}=1.5$ at all wavelengths. We define the focusing efficiency as the energy inside the central lobe over the total illumination energy, the focal length as the length between the external central point of the HFL and the maximum intensity position inside the focal spot.
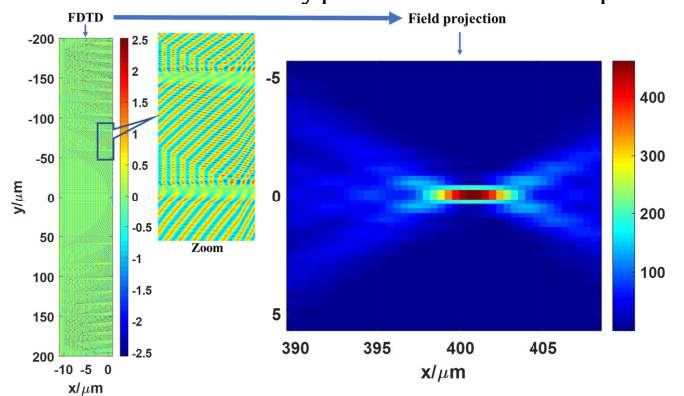

Fig. 3. Intensity distribution computed by FDTD approach combined with ASM field projection for a HFL with $p_{h}=9, \mathrm{~F} / \#=1, \mathrm{D}=400 \mu \mathrm{m}$ and $\lambda=0.5 \mu \mathrm{m}$.
The focus efficiency, focal length, maximum intensity and the full width at half maximum vs wavelength are shown in Fig. 4 for unitary spolarized normal incident plane wave. All the values are given for the maximum intensity position. Three different HFLs with $F / \#=0.5$, $\mathrm{F} / \#=1$ and $\mathrm{F} / \#=3$ have been simulated. A different color corresponds to a different harmonic number $p_{h}$.

Firstly, we notice that the harmonic wavelengths are independent of the F/\#, and approximately fulfill Eq. (3) in all cases. The focal length also approximately verifies Eq. (4). Then, as the design wavelength is $0.5 \mu \mathrm{m}$, we obtain peak efficiency at this wavelength in all cases. In the case where $p_{h}=1$ is an ordinary kinoform lens, we obtain only one peak at $\lambda_{d}$. The focal length monotonically decreases as the wavelength increases, which corresponds to an Abbe number of $v_{\text {refractive }}=-3.5$. If $p_{h}>1$, the number of efficiency peaks increases as $p_{h}$ increases. The FWHM decreases as $\mathrm{F} / \#$ diminishes which corresponds to the diffraction theory results. As we have calculated before, $F / \#=0.5$ is almost the limit for a refractive index $n_{d}=1.5$. However, the FWHM value is larger than $\lambda / 2$ when $F / \#=0.5$ in all cases, which means that a HFL cannot provide super resolution imaging. As the focal length changes with the wavelength, the real $\mathrm{F} / \#$ is also changing with the wavelength. The FWHM almost remains constant for an ordinary Fresnel lens for different wavelengths as shown in Fig. 4(c).

The focus efficiency is a decreasing function of $F / \#$. This is a phenomenon that we cannot obtain from scalar equation Eq. (5). As $\mathrm{F} / \#$ decreases, the radius $r$ increases (see Fig. 2) and the incident angle with respect to the profile of the HFL increases too, which leads to a lower transmittance.

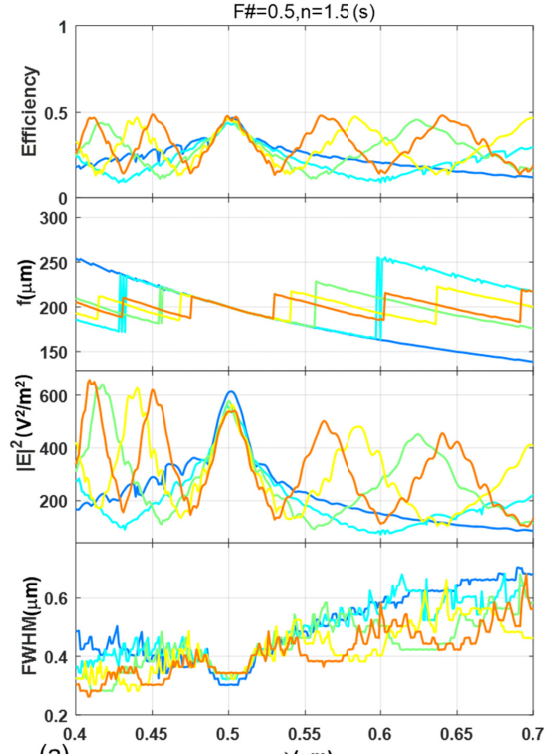

(a)

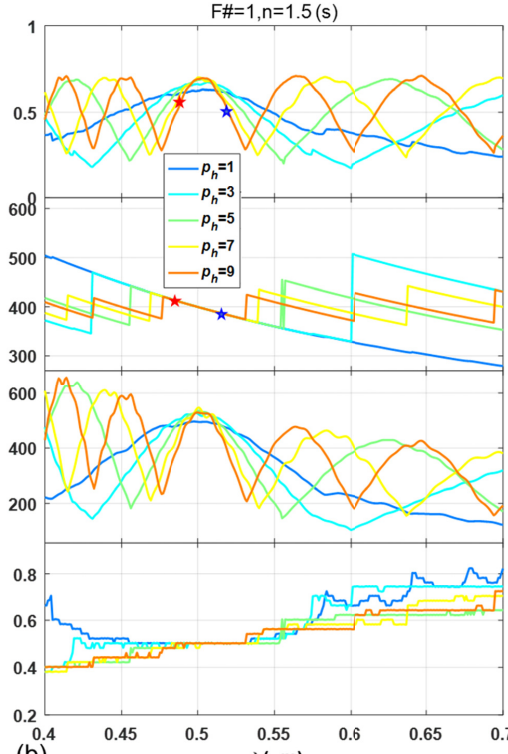

(b)

$\lambda(\mu \mathrm{m})$

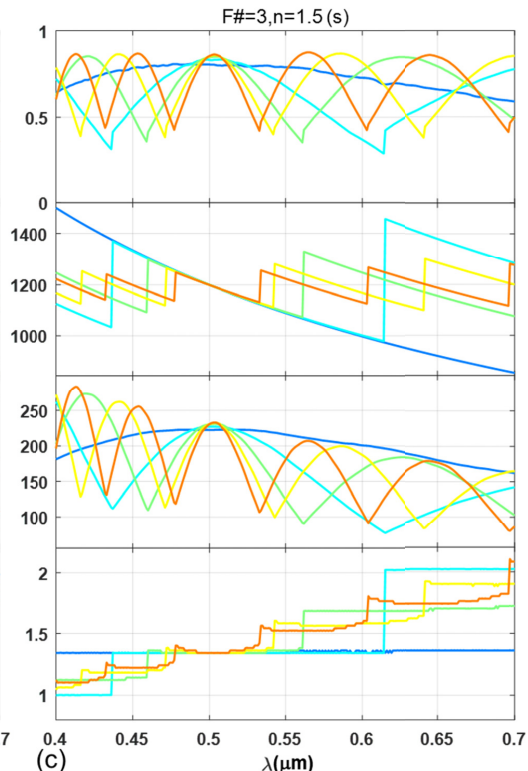

Fig. 4. Optical properties of several HFLs with different F/\# for normal s-polarized plane wave. The focus efficiency, focal length, maximum intensity

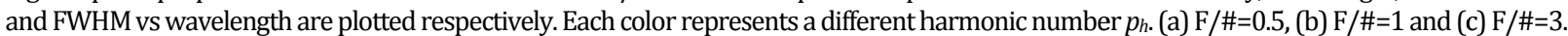

Fig. 4 shows that maximal diffraction efficiencies are obtained at harmonic wavelengths. The discontinuities for focal lengths are obtained at wavelengths where the diffraction efficiencies are minimal. To explain the oscillating phenomenon of the efficiency curve when $p_{h}>1$, we plot electric field intensity distributions in Fig. 5 at two different wavelengths where $p_{h}=9$ and $\mathrm{F} / \#=1$ as an example. The associated results labeled by red and blue stars are pointed out in Fig. 4(b). We observe two clear light spots at each wavelength with different relative intensities. At $\lambda=0.480 \mu \mathrm{m}$, the further spot is stronger, while at $\lambda=0.525 \mu \mathrm{m}$, it is the opposite. At efficiency minima, 
the two light spots have the same intensity, which corresponds to the focal length leaps in Fig. 4.

For the diffraction efficiency and focal length, we compare the paraxial case using Eq. (5) and Eq. (4) with the result obtained by FDTD plus ASM projection as shown in Fig. 6. The calculation method for efficiency in the FDTD plus ASM projection is the energy inside the central lobe over the total illumination energy, which cannot reach 1. However, the maximum efficiency for paraxial approximation can reach 1 at harmonic wavelengths.
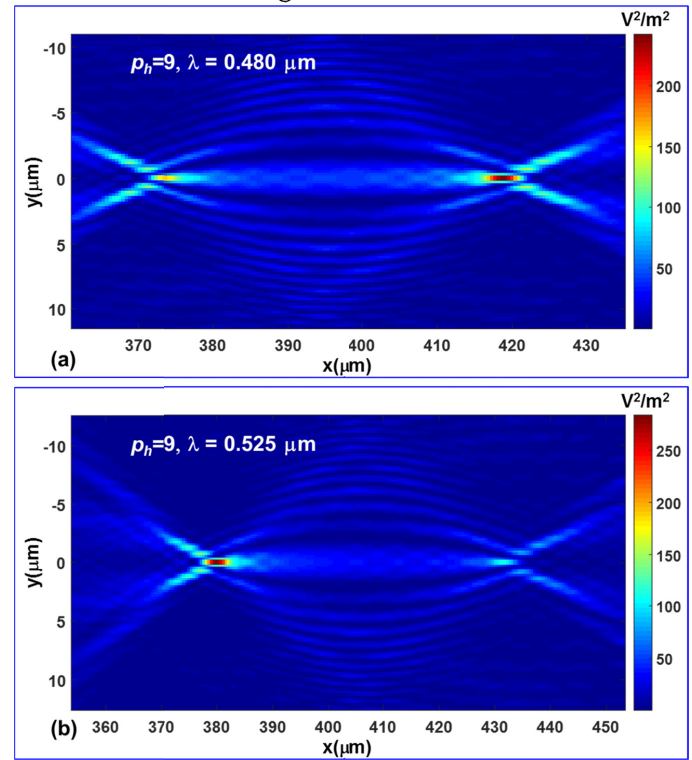

Fig. 5. Electric field intensity distribution of a harmonic lens with $p_{h}=9$ with $F / \#=1$. (a) $\lambda=0.480 \mu \mathrm{m}$ which corresponds to red star in Fig. 4(b) and (b) $\lambda=0.525 \mu \mathrm{m}$ which corresponds to blue star in Fig. 4(b).

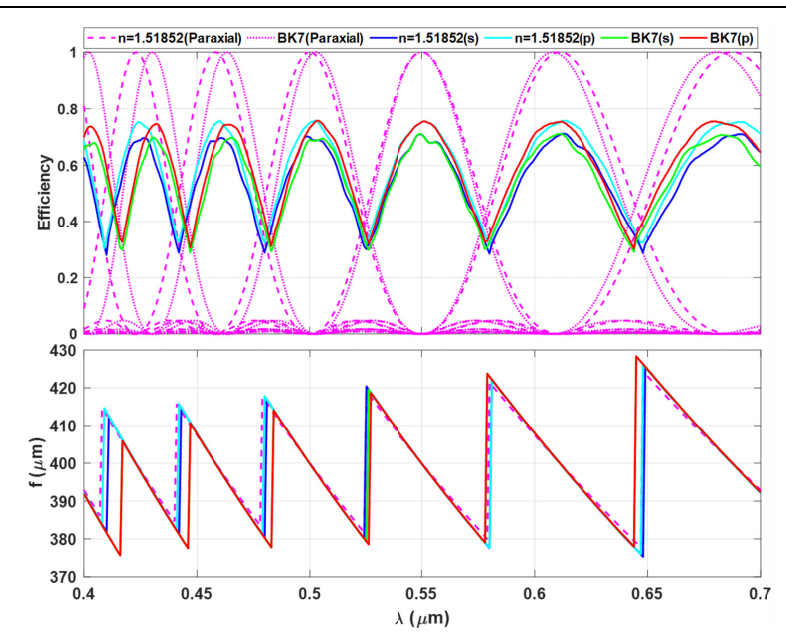

Fig. 6. Comparison of efficiency (top) and focal length (bottom) between the results of paraxial scalar approximation and results calculated by FDTD plus ASM. The harmonic number is $p_{h}=10$, the design wavelength $\lambda_{d}=0.55 \mu \mathrm{m}$ and $\mathrm{F} / \#=1$ in all cases. An ideal material with constant index of refraction $n=1.51852$ and a real material BK7 are considered. Both s and p normal polarized light are simulated.
In Fig. 6, we have also considered the case of a non-dispersive fictitious material with a constant index of refraction $n=1.5185$ which is the index of BK7 at a wavelength of $0.55 \mu \mathrm{m}$ and the case with dispersive glass BK7. The harmonic number is $p_{h}=10$, the design wavelength $\lambda_{d}=0.55 \mu \mathrm{m}$, and $\mathrm{F} / \#=1$ in all cases. Both $s$ and $p$ normal polarizations are considered. Compared to Fig. 4 , we plot efficiency and focal length only because the maximum intensity and FWHM of focal spot are hard to calculate by paraxial approximation.

If we take into account the dispersion of the material, the harmonic wavelengths shift towards the design wavelength $\lambda_{d}$ and the maximum efficiency doesn't change. The values of harmonic wavelengths are almost the same between paraxial approximation and electromagnetic calculation. The shifted points of focal length in each case correspond to the efficiency minima. Considering the polarization, we observe that p-polarized light can have about $5 \%$ higher efficiency than s-polarized light. This difference is mainly caused by the different reflectance on the HFLs surfaces of the two polarizations.

We also calculated the efficiency and focal length of several HFLS using a transmittance function and ASM as shown in Fig. 7. The transmittance function is defined as:

$$
T(r)=\exp \left[j \frac{2 \pi}{\lambda_{d}}(n-1) x_{i}(r)\right],
$$

where $x_{i}(r)$ is calculated by Eq. (7). The harmonic number is $p_{h}=10$, the design wavelength is $\lambda_{d}=0.55 \mu \mathrm{m}$ and the refractive index is $\mathrm{n}=1.51852$. We notice that the harmonic wavelengths are shifted compared to the result calculated by FDTD plus ASM (dashed curve). The deviation gets larger as $\mathrm{F} / \#$ becomes smaller. This study indicates that when considering small F/\# HFLs, FDTD calculation is needed to get accurate result.

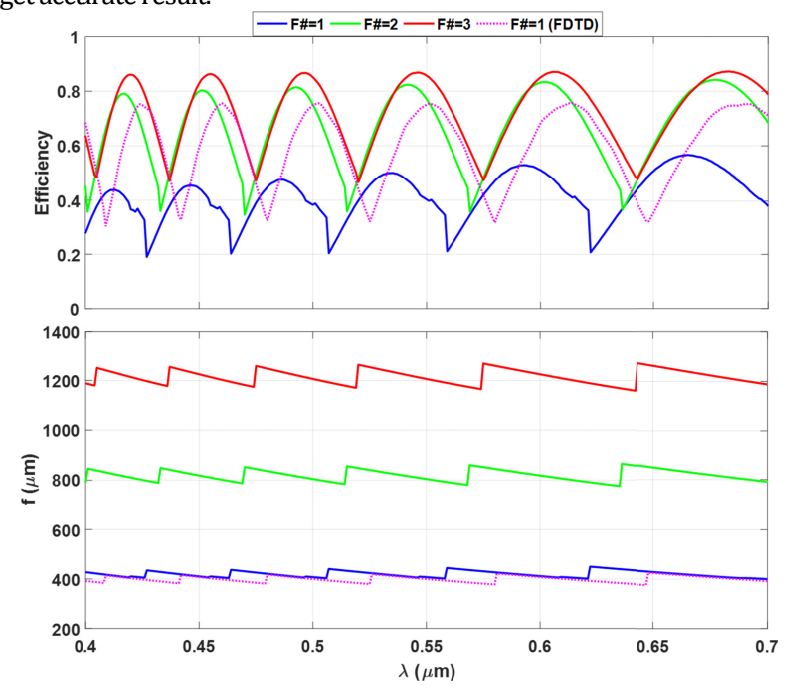

Fig. 7. Efficiency and focal length calculated by using transmittance function and ASM. The harmonic number is $p_{h}=10$, the design wavelength $\lambda_{d}=0.55 \mu \mathrm{m}$. three cases with $\mathrm{F} / \#=1, \mathrm{~F} / \#=2$ and $\mathrm{F} / \#=3$ are plotted. The dashed curve represents the result using FDTD and ASM.

\section{OPTICAL PROPERTIES OF HFLS WITH OBLIQUE ILLUMINATION}

With the FDTD method and the field projection, we have also calculated the case at oblique incidence. In this case, because the determination of the intensity envelope is difficult, due to its nonregularity, we have only calculated the maximum intensity which is 
proportional to efficiency as shown in Fig. 4. We have calculated the maximum intensity of $\mathrm{F} / \#=3$ and $\mathrm{F} / \#=1$ for three different oblique angles $\alpha=1^{\circ}, \alpha=3^{\circ}$, and $\alpha=5^{\circ}$ respectively as shown in Fig. 8. The maximum intensity is obtained within a square area whose side length is $f$ and center is the nominal focus position.
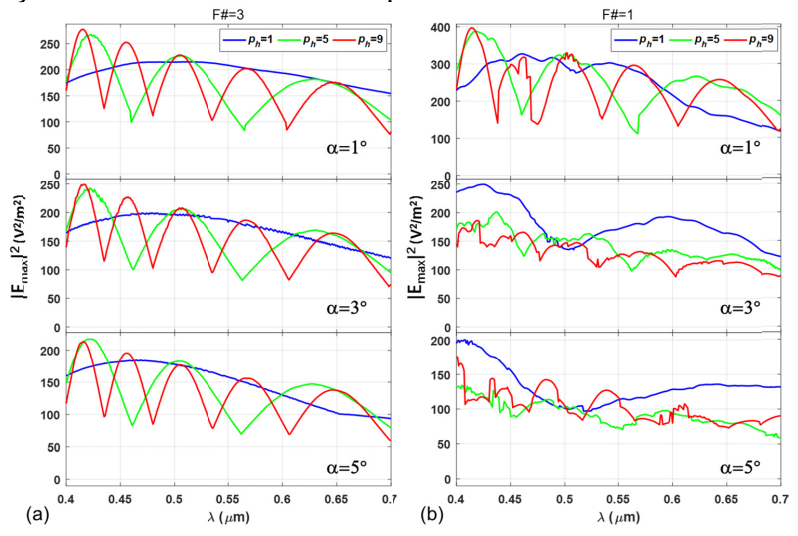

Fig. 8. The maximum electric field intensity vs wavelength with different incident angles $\alpha=1^{\circ}, \alpha=3^{\circ}$ and $\alpha=5^{\circ}$ (a) $\mathrm{F} / \#=3$. (b) $\mathrm{F} / \#=1$. The maximum intensity is obtained within a square area whose side length is $f$ and center is the nominal focus position.

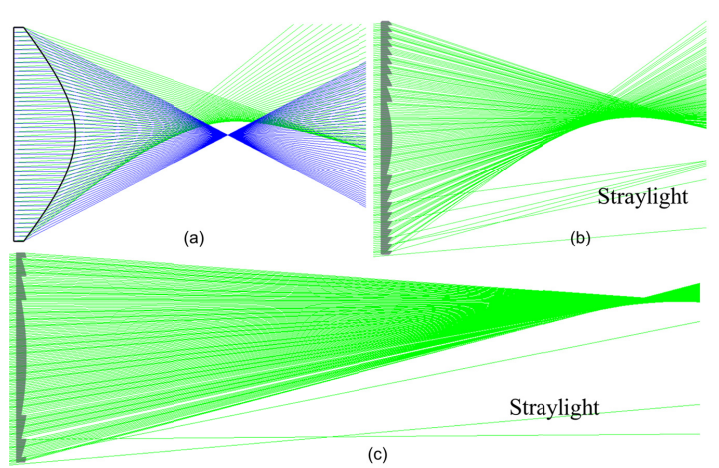

Fig. 9. Raytracing through lenses with design wavelength. (a) Aspherical lens with $\mathrm{F} / \#=1$, raytracing of normal incident rays (blue) and with an oblique incident angle $\alpha=5^{\circ}$ (green). (b) Fresnel lens with $F / \#=1$. Incident angle is $\alpha=5^{\circ}$. (c) Fresnel lens with $F / \#=3$, Incident angle is $\alpha=5^{\circ}$.

The harmonic wavelengths are unchanged if the incident angle is less than a certain value (typically in our example $1^{\circ}$ when $\mathrm{F} / \#=1$ and $5^{\circ}$ when $\mathrm{F} / \#=3$ ). This typical angle value increases as $\mathrm{F} / \#$ increases. The comparison between Fig. 8 and Fig. 4 shows that the maximum intensity decreases much less with the same oblique illumination if $\mathrm{F} / \#$ is larger. We can say that the harmonic wavelengths are more stable when $F / \#$ is larger. If $F / \#$ is smaller, for example $F / \#=1$, the result is more sensitive to the incident angle. We can hardly distinguish which wavelength is the harmonic one at $\alpha=5^{\circ}$.

In geometrical optics, we can obtain the same conclusion using raytracing. The aberrations are more serious if $\mathrm{F} / \#$ is small with the same incident angle as shown in Fig. 9. We also notice that the aspherical lens which can focus plane wave on a perfect point in Fig. 9 (a) has a similar effect to the Fresnel lens in Fig. 9 (b) for the same oblique incidence, except that the Fresnel lens can generate some stray light on the extreme boundary grooves.
Using FDTD and ASM, we plot the electric field intensity distribution of a HFL for $\mathrm{F} / \#=1$ and $p_{h}=1$ with oblique angle $\alpha=5^{\circ}$ at two wavelengths. In Fig. 8, the intensity maximum corresponds to $\lambda=0.4$ $\mu \mathrm{m}$ and the minimum to $\lambda=0.5 \mu \mathrm{m}$. When $p_{h}=9$, the local intensity maximum (respectively minimum) corresponds to $\lambda=0.54 \mu \mathrm{m}$ (respectively $\lambda=0.5 \mu \mathrm{m}$ ). The four electric field intensities are shown in Fig. 10. Because of the aberration, we clearly see caustics which are similar as shown in Fig. 9. Because of the complexity of the caustics, definitions of parameters for evaluation need to be further developed in the future.
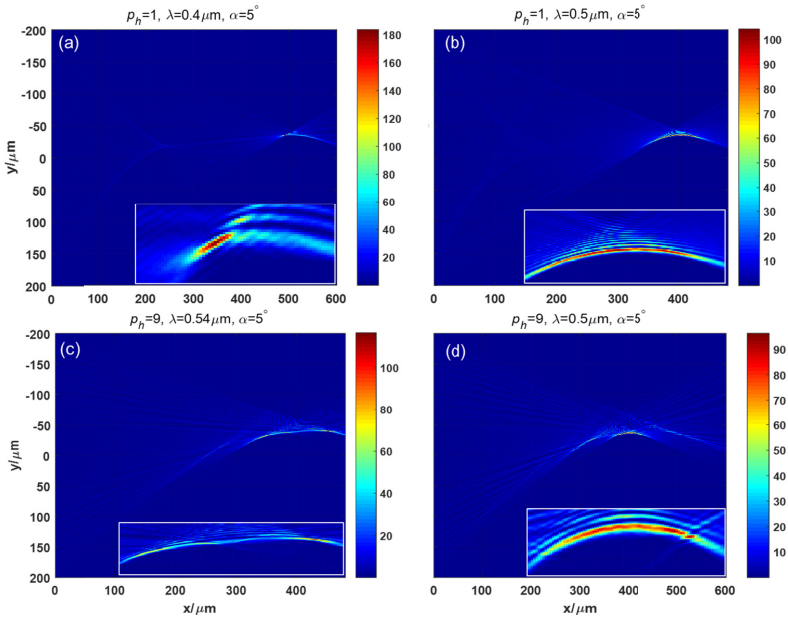

Fig. 10. Electric field intensity distribution of several cases when the illumination angle is $\alpha=5^{\circ}$. The inserts are the zooming in the vicinity of the focal position. $\mathrm{F} / \#=1$ in all the cases. (a) $p_{h}=1, \lambda=0.4 \mu \mathrm{m}$, (a) $p_{h}=1$, $\lambda=0.5 \mu \mathrm{m}$, (a) $p_{h}=9, \lambda=0.54 \mu \mathrm{m}$ and (a) $p_{h}=9, \lambda=0.5 \mu \mathrm{m}$.

\section{CONCLUSION}

HFLs present the advantage of working well with several discrete wavelengths for normal incidence. We have investigated the efficiency, focal length, maximum focus intensity and FWHM of the focal spot for several harmonic numbers and F-numbers using FDTD and ASM field projection. Compared to the paraxial approximation, the values of the harmonic wavelengths are almost the same both with constant refractive index and dispersive materials. Moreover, a FDTD analysis can give more detailed information. We can summarize our main results in the following statements. Considering F/\#, the harmonic wavelengths do not change with $\mathrm{F} / \#$; the diffraction efficiency and FWHM of the focus are increasing functions of F/\#. For the polarization, we observe that p-polarized light has higher efficiency than s-polarized light (from our examples the difference is typically of $5 \%$ ). When considering the dispersion of real optical glasses, the harmonic wavelengths shift towards the designed wavelength but do not change the maximum efficiencies. Finally, for oblique illumination, we conclude that the diffraction efficiency decreases as the oblique angle of incidence increases because of the aberrations. With larger $\mathrm{F} / \#$, HFLs can stand larger angles of incidence, and the harmonic wavelengths are also unchanged with different angle of incidence if the aberrations are not too high. In the next step, quantitative methods should be developed to study the off-axis aberrations of the HFLs. Meanwhile, it would be very useful if a raytracing model for HFLs could be built and incorporated into optical design software such as Zemax and Code V. Finally, the experimental verification should be carried out. 


\section{References}

1. L. Lesem, P. Hirsch, and J. Jordan, "The kinoform: a new wavefront reconstruction device," IBM J. Res. Devel. 13, 150-155 (1969).

2. J. Jordan, P. Hirsch, L. Lesem, and D. Van Rooy, "Kinoform lenses," Appl. Opt. 9, 1883-1887 (1970).

3. T. Stone and N. George, "Hybrid diffractive-refractive lenses and achromats," Appl. Opt. 27, 2960-2971 (1988).

4. B. H. Kleemann, M. Seesselberg, and J. Ruoff, "Design concepts for broadband high--efficiency DOEs," J. Eur. Opt. Soc. Rapid Publ. 3, 08015 (2008).

5. S. Mao, Q. Cui, M. Piao, and L. Zhao, "High diffraction efficiency of threelayer diffractive optics designed for wide temperature range and large incident angle," Appl. Opt. 55, 3549-3554 (2016).

6. M. Piao, Q. Cui, H. Zhu, C. Xue, and B. Zhang, "Diffraction efficiency change of multilayer diffractive optics with environmental temperature," Journal of Optics 16, 035707 (2014).

7. J. Wang and C. Xue, "Athermalization and thermal characteristics of multilayer diffractive optical elements," Appl. Opt. 54, 9665-9670 (2015).

8. H. Yang and C. Xue, "Diffraction efficiency evaluation for diamond turning of multilayer diffractive optical elements," Opt. Eng. 56, 075101 (2017).

9. D. W. Sweeney and G. E. Sommargren, "Harmonic diffractive lenses," Appl. Opt. 34, 2469-2475 (1995).

10. D. Faklis and G. M. Morris, "Spectral properties of multiorder diffractive lenses," Appl. Opt. 34, 2462-2468 (1995).

11. B. Groever, W. T. Chen, and F. Capasso, "Meta-Lens Doublet in the Visible Region," Nano Lett. 17, 4902-4907 (2017).

12. E. Arbabi, A. Arbabi, S. M. Kamali, Y. Horie, and A. Faraon, "Multiwavelength polarization-insensitive lenses based on dielectric metasurfaces with meta-molecules," Optica 3, 628-633 (2016).

13. A. Arbabi, Y. Horie, A. J. Ball, M. Bagheri, and A. Faraon, "Subwavelengththick lenses with high numerical apertures and large efficiency based on high-contrast transmitarrays," Nat. Commun. 6, 7069 (2015).

14. F. Aieta, M. A. Kats, P. Genevet, and F. Capasso, "Multiwavelength achromatic metasurfaces by dispersive phase compensation," Science 347, 1342-1345 (2015).

15. F. Aieta, P. Genevet, M. A. Kats, N. Yu, R. Blanchard, Z. Gaburro, and F. Capasso, "Aberration-free ultrathin flat lenses and axicons at telecom wavelengths based on plasmonic metasurfaces," Nano Lett. 12, 49324936 (2012).

16. S. S. Lane, M. Morris, L. Nordan, M. Packer, N. Tarantino, and R. B. Wallace, "Multifocal intraocular lenses," Ophthalmology Clinics 19, 89-105 (2006).

17. H. C. Hunt and J. S. Wilkinson, "Kinoform microlenses for focusing into microfluidic channels," Opt. Express 20, 9442-9457 (2012).

18. S. H. Yan, J. Zhang, C. L. Zhou, and Y. Xu, "Research on Dispersion Performance of Harmonic Diffractive Lenses," Journal of Physics: Conference Series 48, 897-901 (2006).

19. J. M. Bendickson, E. N. Glytsis, and T. K. Gaylord, "Scalar integral diffraction methods: unification, accuracy, and comparison with a rigorous boundary element method with application to diffractive cylindrical lenses," J. Opt. Soc. Am. A 15, 1822-1837 (1998)

20. J. M. Bendickson, E. N. Glytsis, and T. K. Gaylord, "Metallic surface-relief on-axis and off-axis focusing diffractive cylindrical mirrors," J. Opt. Soc. Am. A 16, 113-130 (1999).

21. J.-S. Ye, B.-Y. Gu, S.-T. Liu, and B.-Z. Dong, "Applications of improved first Rayleigh-Sommerfeld method to analyze the performance of cylindrical microlenses with different f-numbers," J. Opt. Soc. Am. A 22, 862-869 (2005).

22. B.-Z. Dong, J.-S. Ye, J. Liu, B.-Y. Gu, G.-Z.Yang, M.-H. Lu, and S.-T. Liu, "Analysis of the focal characteristics of cylindrical lenses made of anisotropically dielectric material based on rigorous electromagnetic theory," J. Mod. Opt. 50, 1195-1208 (2003).

23. J. Lin, J. Ye, and S. Liu, "Focusing performance of the closed-boundary cylindrical microlenses analyzed by the boundary element method," Opt. Commun. 266, 25-31 (2006).

24. J.-S. Ye and Y. Zhang, "Rigorous electromagnetic analysis of metallic cylindrical focusing micromirrors with high diffraction efficiency, achromatic aberration and long focal depth," Opt. Commun. 283, 16611667 (2010).

25. J.-S. Ye, Y. Zhang, and K. Hane, "Improved first Rayleigh-Sommerfeld method applied to metallic cylindrical focusing micro mirrors," Opt. Express 17, 7348-7360 (2009).

26. J.-S. Ye, B.-Y. Gu, B.-Z. Dong, and S.-T. Liu, "Improved first RayleighSommerfeld method for analysis of cylindrical microlenses with small $\mathrm{f}$ numbers," Opt. Lett. 29, 2345-2347 (2004).

27. A. Albarazanchi, P. Gérard, P. Ambs, P. Meyrueis, G.-N. Nguyen, and K. Heggarty, "Smart multifunction diffractive lens experimental validation for future PV cell applications," Opt. Express 24, A139-A145 (2016).

28. F. Di, Y. Yingbai, J. Guofan, and W. Minxian, "Rigorous concept for the analysis of diffractive lenses with different axial resolution and high lateral resolution," Opt. Express 11, 1987-1994 (2003).

29. J. Jiang and G. P. Nordin, "A rigorous unidirectional method for designing finite aperture diffractive optical elements," Opt. Express 7, 237-242 (2000).

30. S. D. Mellin and G. P. Nordin, "Limits of scalar diffraction theory and an iterative angular spectrum algorithm for finite aperture diffractive optical element design," Opt. Express 8, 705-722 (2001). 